\title{
Neogenin-1 Promotes Cell Proliferation, Motility, and Adhesion by Up-Regulation of Zinc Finger E-Box Binding Homeobox 1 Via Activating the Rac1/PI3K/AKT Pathway in Gastric Cancer Cells
}

\author{
Hengyi Qu Huabo Sun Xueping Wang
}

Department of Gastroenterology, Yantai Affiliated Hospital of Binzhou Medical University, Yantai, China

\section{Key Words}

Gastric cancer • Neogenin-1 • Epithelial mesenchymal transition • Zinc finger E-box-binding homeobox $1 \cdot \operatorname{Rac} 1 /$ PI3K/AKT

\begin{abstract}
Background/Aims: Neogenin-1 (Neo1) has been reported to be involved in diverse physiology and pathology functions, including cell proliferation, differentiation and migration. The present study aimed to explore the functional role of neogenin-1 (Neo1) in gastric cancer (GC), together with underlying mechanisms. Methods: Neo1 expression was analyzed by qRT-PCR and Western blot analysis in both human GC cell lines and normal gastric epithelial cell line. Neo1 was respectively overexpressed or silenced by transfection with pCDNA3.1 or siRNA, and then the cells were incubated with or without different concentrations of cisplatin, transforming growth factor (TGF)- $\beta 1$, and/or inhibitors of Rac- 1 and PI3K. Thereafter, cell viability, invasion, and adhesion were measured by CCK-8, wound healing and adhesion assays, respectively. The expression levels of key factors involved in epithelial mesenchymal transition (EMT) and the PI3K/AKT pathway were analyzed by Western blot analysis. Results: The results showed that the Neo1 level was significantly increased in GC cell lines, with the highest level in SGC-7901 cells. Overexpression of Neol significantly reduced the GC cell sensitivity to cisplatin and increased the cell viability, motility and adhesion ability, and while silencing of $\mathrm{NeO} 1$ showed contrary results. Moreover, overexpression of Neol dramatically downregulated the $\mathrm{E}$-Cadherin level and upregulated the levels of $\mathrm{N}$-Cadherin and Vimentin. In addition, the data revealed that Neo1 positively regulated the expression of Zinc finger E-box-binding homeobox 1 (ZEB1) by activating the Rac1/PI3K/AKT pathway. Conclusions: Neo1 could promote cell proliferation, motility, and adhesion by up-regulation of ZEB1 via activating the Rac1/PI3K/AKT pathway in GC cells.

H. Qu and H. Sun contributed equally to this work.

\begin{tabular}{ll}
\hline Hengyi Qu & Department of Gastroenterology, Yantai Affiliated Hospital of Binzhou Medical University \\
& No. 717, Jinfu Street, Yantai 264100, Shandong (China) \\
E-Mail qihengyi0322@126.com
\end{tabular}
\end{abstract}




\section{Introduction}

Gastric cancer (GC) has been reported as the fourth most common kind of cancer and the second most leading cause of cancer-related deaths globally [1]. Although tremendous progress has been made in recent years in diagnostic techniques and perioperative management of GC, there were about 986, 600 new GC cases and 738, 000 deaths in 2008 worldwide [1]. Currently, the available treatments of GC include surgery, chemotherapy, radiation therapy, or a combination of modalities. However, the prognosis of GC is still dismal due to the usually late diagnosis and high rate of recurrence [2]. Even the patients who have received curative surgical resection often die from recurrent GC, distant metastases and/or drug resistance [3]. Therefore, there is an urgent need to explore the underlying molecular mechanisms of GC cell growth, metastasis and drug resistance.

Neogenin-1 (Neo1) is a multifunctional transmembrane receptor, which belongs to the immunoglobulin superfamily [4]. Interestingly, it has been reported that Neo1 shares $50 \%$ amino-acid identity with the tumor suppressor molecule deleted in colon cancer (DCC) [4]. Neo1 is observed in the tissues where active growth occurs and it is also involved in tissue growth, migration, cell-cell adhesion, cell-cell recognition, cell proliferation and differentiation $[4,5]$. Abnormal expression of Neo1 was found in many human tumors such as glioma, medulloblastoma, esophageal squamous cell carcinoma (ESCC), breast cancer, pancreas cancer, cervix cancer, and colorectal cancer [6-13]. Recently, up-regulation of Neo1 was observed in GC tissues and cell lines, and elevated expression of Neo1 was responsible for the GC cell proliferation and migration [14]. However, the role of Neo1 in GC remains uncertain due to its complicated functions [4].

It has been well acknowledged that epithelial mesenchymal transition (EMT) is implicated in cancer progression, particularly in the early steps of metastasis [15]. EMT is a biological process in which epithelial cell layers lose polarity and cell-cell interactions and undergo multiple biochemical changes [16]. EMT could promote migratory capacity and motility, increase resistance to apoptosis, and significantly elevate production of extracellular matrix (ECM) [17]. Both Neo1 and EMT play significant roles in tumor metastasis, however, little information is available regarding the potential relationship between Neo1 and EMT.

Therefore, in the present study, we sought to investigate the functional role of Neo1 in GC and explore the regulatory mechanism of Neo1 on EMT, together with underlying potential cell signaling pathway. Our study might provide a new insight into treatment of GC.

\section{Materials and Methods}

\section{Cell culture and treatment}

Human GC cell lines including MKN-28, BGC-823, MGC-803, SGC-7901 and MKN-45, and human normal gastric epithelial cell line GES-1 were all obtained from Cell bank of Chinese Academy of Sciences (Shanghai, China). These cells were maintained in RPMI 1640 (Sigma-Aldrich, St. Louis, MO, USA) supplemented with $10 \%$ fetal bovine serum (FBS; Sigma-Aldrich), 100 units/ml penicillin (GIBCO, Grand Island, NY, USA), and $100 \mu \mathrm{g} / \mathrm{ml}$ streptomycin (GIBCO) in a humidified atmosphere of $5 \% \mathrm{CO}_{2}$ at $37^{\circ} \mathrm{C}$. Transforming growth factor (TGF)- $\beta 1$ (10 ng/ml; Sigma-Aldrich) was used to induce the EMT in GC cells. Cells were incubated in

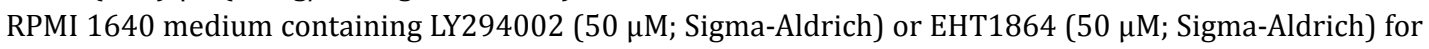
$24 \mathrm{~h}$ in order to inhibit PI3K/AKT or Rac1.

\section{Plasmids and siRNA transfection}

Neo1 expression vector (pc-Neo1) was constructed by sub-cloning the full-length wild-type Neo1 coding sequence into pcDNA3.1 (+), and was confirmed by sequencing. The empty construct pcDNA3.1 was considered as a control. The target sequence for Neo1-specific small interfering RNA (si-Neo1; 5'-AGAUCUGGA GGUUUCACAUCUUU GG-3') and control siRNA (no silencing; siNC) were synthesized by GenePharma Co. (Shanghai, China). Cell transfection was conducted using Lipofectamine 3000 reagent (Invitrogen, Carlsbad, CA, USA) following the manufacturer's protocol. 


\section{Cellular Physiology Cell Physiol Biochem 2018;48:1457-1467 and Biochemistry Published $\begin{aligned} & \text { DOI: 10.1159/000492255 } \\ & \text { (c) } 2018 \text { The Author(s). Published by S. Karger AG, Basel } \\ & \text { www.karger.com/cpb }\end{aligned}$}

Qu et al.: Neogenin-1 Acts as An Oncogene in GC

Cell viability and cell survival

The effects of Neo1 abnormal expression on cell viability and cell survival rate were determined by Dojindo Cell Counting kit-8 (CCK-8; Dojindo Molecular Technologies, Kumamoto, Japan). Briefly, SGC-7901 cells were seeded in a 96-well plate $\left(5 \times 10^{3}\right.$ cells/well), and then the cells were transfected with pc-Neo1, si-Neo1, or their corresponding controls. For the cell survival rate, the cells were incubated with or without different concentrations of cisplatin $(0,50,100,150$, and $200 \mu \mathrm{M})$ after transfection. Subsequently, the CCK8 solution was added to the culture medium, and then cells were incubated for $1 \mathrm{~h}$ at $37^{\circ} \mathrm{C}$ in humidified $95 \%$ air and $5 \% \mathrm{CO}_{2}$. Absorbance at $450 \mathrm{~nm}$ was measured using a Microplate Reader (DTX 800, Beckman, CA, USA).

\section{Wound healing assay}

The effects of $\mathrm{Neo} 1$ abnormal expression on cell migration were evaluated using in vitro wound-healing model according to the manufacturer's instructions. In brief, SGC-7901 cells were plated in 6-well plates (1 $\times 10^{4}$ cells/well) and incubated overnight. After transfection with pc-Neo1, si-Neo1, or their corresponding controls, wounds were created by scratching cell sheets with a sterile $200 \mu$ l pipette tip. Debris was removed from the wound and the edges were smoothened out with phosphate buffer solution (PBS). Photographs were acquired at $24 \mathrm{~h}$ by an inverted microscope (IX83, Olympus, Tokyo, Japan). The wound closure was quantitatively analyzed by Image J software (National Institutes of Health, Bethesda, MD, USA) by circling and assessing the unhealed area in the wound images. Each test was repeated in triplicate.

\section{Adhesion assay}

The effects of Neo1 abnormal expression on cell adhesion ability was analyzed by an adhesion assay according to the manufacturer's instructions. Briefly, after transfection with pc-Neo1, si-Neo1, or their corresponding controls, the SGC-7901 cells were trypsinized, suspended in $200 \mu \mathrm{l}$ of serum-free medium and added to Matrigel-coated inserts (8- $\mu \mathrm{m}$ pore size; BD Bioscience, Franklin Lakes, NJ, USA). These cells were maintained in RPMI 1640 medium supplemented with 2\% FBS. After twenty-four hours, the cells were removed from the upper surface of the membrane by a cotton-tipped swab. Thereafter, the membranes were fixed with $4 \%$ paraformaldehyde for $10 \mathrm{~min}$ and mounted over slides with $5 \mu \mathrm{l}$ of Fluoromount reagent with 4, 6-diamidino-2-phenylindole (DAPI; Sigma-Aldrich).

\section{Quantitative real-time reverse transcriptase polymerase chain reaction (qRT-PCR)}

Total RNA was isolated using TRIzol (Invitrogen) according to the manufacturer's instructions. RNA was reversely transcribed to complementary DNA (cDNA) using PrimeScriptTM RT Master Mix (Takara, Otsu, Japan). PCR specific primers were designed by GenePharma. The primers were listed as following: Neo1 Forward: 5'-AAATA GTCAA CAAGC ATGGT AAAAA-3' and Reverse: 5'-AAGAC ATGTC CACAG CCACA-3'; zinc finger E-box-binding homeobox 1 (ZEB1) Forward: 5'-GATGA CCTGC CAACA GACCA-3' and Reverse: 5'-CCCCA GGATT TCTTG CCCTT-3'; GAPDH Forward: 5'-GCACC GTCAA GGCTG AGAAC-3' and Reverse: 5'-TGGTG AAGAC GCCAG TGGA-3'. qRT-PCR was performed on the Bio-Rad CFX 96 Real-time PCR system (Bio-Rad, Hercules, CA) using SYBR ${ }^{\circledast}$ Premix Ex TaqTM II (TaKaRa, Shiga, Japan) according to the manufacturer's instructions. The mRNA level was normalized to GAPDH using the comparative $2^{-(\Delta \triangle C q)}$ method. PCR conditions were performed as the following parameters: 1 predenaturation cycle of $5 \mathrm{~min}$ at $94^{\circ} \mathrm{C}, 35$ cycles of denaturation at $94^{\circ} \mathrm{C}$ for $15 \mathrm{~s}, 59^{\circ} \mathrm{C}$ for $45 \mathrm{~s}$, and $72^{\circ} \mathrm{C}$ for $2 \mathrm{~min}$, and a final extension at $72^{\circ} \mathrm{C}$ for $5 \mathrm{~min}$.

\section{Western blot}

After transfection and/or treatment, the cell suspension was collected and centrifuged. Total protein was extracted from the cells using RIPA lysis buffer (Beyotime Biotechnology, Shanghai, China). The protein concentration was quantified using a BCA ${ }^{\mathrm{TM}}$ Protein Assay Kit (Thermo Scientific, Pierce Protein Research Products, Rockford, IL, USA). Equal amount of protein (20 $\mu$ g per lane) was subjected to $10-12 \%$ sodium dodecyl sulfate (SDS)-polyacrylamide gel electrophoresis (PAGE) gels. The protein was then transferred onto Hybond ECL $0.45 \mu \mathrm{m}$ nitrocellulose membranes (Amersham, Piscataway, NJ, USA). Primary antibodies were prepared in $5 \%$ blocking buffer and incubated with following primary antibodies overnight at $4^{\circ} \mathrm{C}$ : anti-Neo1 antibody (ab183511; Abcam, Cambridge, UK), anti-E-Cadherin antibody (\#3195; Cell signaling Technology, Danvers, MA, USA), anti-N-Cadherin antibody (\#13116; Cell signaling Technology), 


\section{Cellular Physiology Cell Physiol Biochem 2018;48:1457-1467 \begin{tabular}{l|l} 
DOI: 10.1159/000492255 & $\begin{array}{l}\text { O 2018 The Author(s). Published by S. Karger AG, Basel } \\
\text { www.karger.com/cpb }\end{array}$
\end{tabular} \\ Qu et al.: Neogenin-1 Acts as An Oncogene in GC}

anti-Vimentin antibody _ (\#5741; Cell signaling Technology), anti-ZEB1 antibody (\#3396; Cell signaling Technology), anti-guanosine-5'-triphosphate (GTP)-Rac1 antibody (\#26903; NewEast Biosciences, Malvern, PA, USA), anti-Rac1 antibody (\#10101; NewEast Biosciences), anti-phospho (p)-phosphoinositide 3-kinase antibody (p-PI3K; \#4228; Cell signaling Technology), anti-PI3K antibody (\#4249; Cell signaling Technology), anti-AKT antibody (\#9272; Cell signaling Technology), or anti-p-AKT antibody (\#4060; Cell signaling Technology). GAPDH (ab9485; Abcam) was used as a loading control. Membranes were then washed three times with Tris Buffered Saline with Tween (TBST) buffer and incubated with the goat antirabbit secondary antibody (ab6721; Abcam) marked by horseradish peroxidase (Cell signaling Technology) for $2 \mathrm{~h}$ at room temperature. Signals were acquired with WEST-ZOL-plus Western Blot Detection System (INtRON Biotechnology, Sungnam, Korea) and was quantified using Image Lab ${ }^{\text {TM }}$ Software (Bio-Rad).

\section{Statistical analysis}

All experiments were repeated at least three times. Data are presented as the mean \pm standard deviation (SD). Statistical analyses were performed using the Statistics Package for Social Science 19.0 (SPSS Inc., Chicago, IL, USA). The $P$-values were calculated using a one-way analysis of variance (ANOVA). A $P$ value of $<0.05$ was considered statistically significant.

\section{Results}

\section{Expression of Neo1 in GC cell lines}

To investigate the functional role of Neo1 in GC, we first analyzed the expression of Neo1 in GC cell lines. The expression of Neo1 was analyzed by both qRT-PCR and Western blot. As shown in Fig. 1A-1B, both the mRNA and protein levels of Neo1 were significantly increased in GC cell lines MKN-28 $(P<0.05$ for both mRNA and protein), BGC-823 $(P<0.05$ for both mRNA and protein), MGC-803 $(P<0.05$ for both mRNA and protein), SGC-7901 $(P<0.01$ for mRNA and $P<0.001$ for protein) and MKN-45 ( $P<0.05$ for mRNA and $P<0.01$ for protein) compared to the normal gastric epithelial cell line GES-1. In addition, we found that the levels of Neo1 were the highest in SGC-7901 cells. Therefore, we chose SGC-7901 cells for further experiment. Then the cell viability, migration and invasion of SGC-7901 cells were assessed. From Fig. 1C-E, we observed that SGC-7901 cells revealed significantly high cell viability $(P<$ 0.01 at 2 and 3 days; $P<0.001$ at 4 days), migration $(P<0.01$ at 2 days; $P<0.001$ at 3 and 4 days), and adhesion ability $(P<0.01)$ compared to the normal GES- 1 cells.

\section{Effects of Neo1 abnormal expression on GC cell sensitivity to cisplatin}

We then analyzed the effects of abnormal expression of Neo1 on GC cell sensitivity to cisplatin. Neo1 were overexpressed or suppressed by transfection with pc-Neo1 or si-Neo1, respectively. The transfection efficiencies were confirmed by Western blot. As indicated in Fig. 2A, the results showed that the protein levels of Neo1 were significantly increased by transfection with pc-Neo1 but dramatically decreased by transfection with si-Neo1 compared to their corresponding controls (both $P<0.001$ ). The results implied high transfection efficiencies. The survival rate of SGC-7901 cells exposed to different concentrations of cisplatin $(0,50,100,150$, or $200 \mu \mathrm{M})$ was analyzed by CCK-8 assay after transfection. As expected, the percentages of cell survival rate were significantly reduced by cisplatin in a concentration-dependent manner (Fig. 2B). In addition, we observed that the percentages of cell survival rate were statistically upregulated by overexpression of Neo1 compared to the pc-DNA3.1 group $(P<0.05$ for 100,150 , and $200 \mu \mathrm{M})$. In contrast, we found that silencing of Neo1 upregulated the GC cell sensitivity to cisplatin $(P<0.05$ for 100,150 and $200 \mu \mathrm{M})$. These results indicated that high expression of Neo1 reduced the GC cell sensitivity to cisplatin. 
Fig. 1. Expression levels of Neo1 in GC cell lines. Both mRNA and protein levels of Neo1 were respectively measured by qRT-PCR and Western blot in both GC cell lines (MKN-28, BGC-823, MGC803, SGC-7901 and MKN-45) and normal gastric epithelial cell line. The results showed that the both the mRNA (A) and protein (B) levels of Neo1 were significantly increased in GC cell lines compared to the normal epithelial cell line. In addition, the results showed that GC SGC-7901 cells revealed significantly high cell viability (C) and metastasis

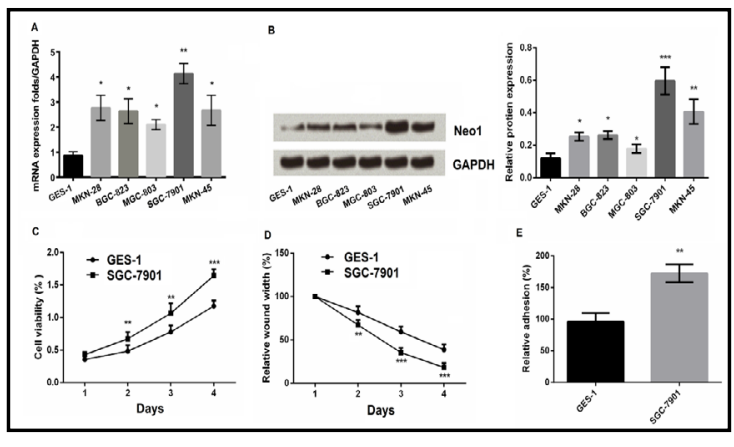
(D-E) compared to the normal GES-1 cells. The experiments were repeated at least three times. Data are presented as the mean \pm standard deviation (SD). Neo1, neogenin-1; GC, gastric cancer; qRT-PCR, quantitative real-time reverse transcriptase polymerase chain reaction. ${ }^{*} \mathrm{P}<0.05,{ }^{, *} \mathrm{P}<0.01$, or ${ }^{* * *} \mathrm{P}<0.001$.

Fig. 2. Effects of Neo1 abnormal expression on GC cell sensitivity to cisplatin. Neo1 was overexpressed or downregulated by transfection with pc-Neo1 or si-Neo1 in SGC7901 cells, respectively. The transfection efficiencies were analyzed by Western blot. After

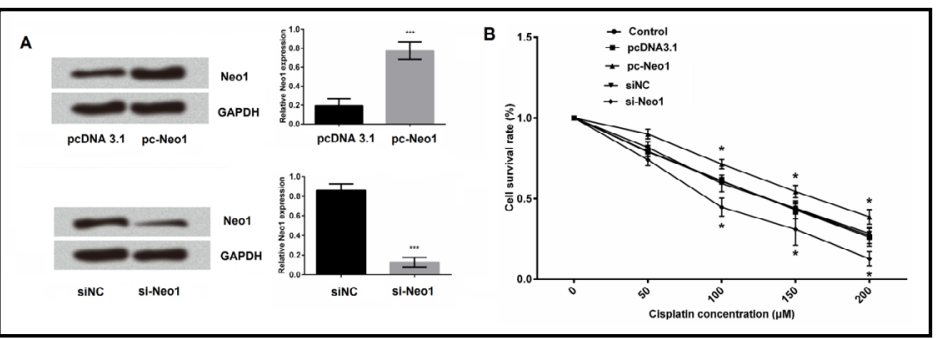
transfection, the survival rate of SGC-7901 cells exposed to different concentrations of cisplatin $(0,50,100,150$, and $200 \mu \mathrm{M})$ was analyzed by CCK-8 assay. The results revealed high transfection efficiency. The protein expression of Neo1 was significantly increased by pc-Neo1 and suppressed by si-Neo1 (A) and GC cell sensitivity to cisplatin was markedly reduced by overexpression of Neo1 and was statistically increased by downregulation of Neo1 in a dose-dependent manner (B). The experiments were repeated at least three times. Data are presented as the mean \pm standard deviation (SD). Neo1, neogenin-1; GC, gastric cancer; pc-Neo1, Neo1 expression vector; si, small interfering; $\mathrm{NC}$, negative control; CCK-8, cell counting kit-8. ${ }^{*} \mathrm{P}<0.05$, or ${ }^{* * *} \mathrm{P}<0.001$.

\section{Effects of Neo1 overexpression on GC cell viability, cell motility and adhesion ability}

Subsequently, we explored the effects of Neo1 overexpression on GC cell viability, cell motility and adhesion ability, together with underlying mechanisms. As demonstrated in Fig. 3A-3C, overexpression of Neo1 significantly promoted cell viability, cell motility and adhesion ability in GC cells (all $P<0.05$ ). The potential underlying mechanism was further investigated. It has been well demonstrated that EMT is involved in cancer progression, particularly in metastasis. In combination of the results from Fig. 1 and Fig. 2, we assumed that there might be a potential relationship between Neo1 and EMT process. Therefore, both the mRNA and protein levels of EMT related factors (E-Cadherin, N-Cadherin, and Vimentin) were measured under the condition of Neo1 abnormal expression. The EMT process of GC cells was induced by administration of TGF- $\beta 1$. The results showed that TGF- $\beta 1$ statistically decreased expression of E-Cadherin $(P<0.01$ for both mRNA and protein), and while markedly increased expression of $\mathrm{N}$-Cadherin (both mRNA and protein) and Vimentin ( $P$ $<0.01$ for mRNA and $P<0.05$ for protein), indicating the EMT was successfully induced. Interestingly, we found that overexpression of Neo1 could significantly decrease the expression of E-Cadherin $(P<0.05$ for mRNA and $P<0.01$ for protein), and while statistically increased the expression of N-Cadherin and Vimentin $(P<0.05$ for both mRNA and protein), as compared to the pcDNA3.1 group. Similarly, the effects of Neo1 overexpression on EMT in cells treated with TGF- $\beta 1$ were identical to that described above $(P<0.05$ or $P<0.01)$ (Fig. 3D and 3F). The results implied that the increasing of cell motility and adhesion ability induced by overexpression of Neo1 was possibly associated with the enhanced EMT. 
Fig. 3. Effects of Neo1 overexpression on GC cell viability, cell motility and adhesion ability. Neo1 was overexpressed by transfection with pc-Neo1 in SGC-7901 cells, and then the effects of Neo1 overexpression on the cell viability, cell motility and adhesion ability were determined by CCK- 8 assay, wound healing assay, and adhesion assay, respectively. The results showed that overexpression of Neo1 significantly increased the cell viability (A), cell motility (B), and adhesion ability (C). The potential underlying mechanisms of cell motility and adhesion ability were further explored by analyzing the EMT process. EMT was constructed by addition of TGF- $\beta 1$ to the cells. Both the mRNA and proteins levels of the key proteins in the EMT process (E-Cadherin, N-Cadherin, and Vimentin) were measured by qRT-PCR and Western blot, respectively. The results indicated that overexpression of Neo1 promoted and EMT process (D-F) in GC cells. The experiments were repeated at least three times. Data are presented as the mean \pm standard deviation (SD). Neo1, neogenin-1; GC, gastric cancer; TGF, transforming growth factor; EMT, epithelial mesenchymal transition; qRT-PCR, quantitative real-time

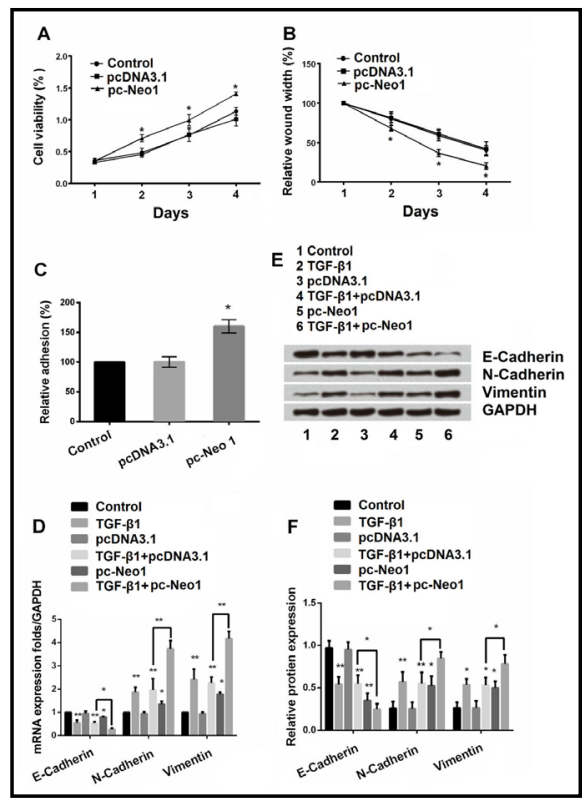
reverse transcriptase polymerase chain reaction; pc-Neo1, Neo1 expression vector. Asterisk for the TGF- $\beta 1$ group indicated significance compared with control; Asterisk for the TGF- $\beta 1+$ pcDNA3.1 and the pc-Neo1 groups indicated significance compared with the pcDNA3.1 group. ${ }^{*} \mathrm{P}<0.05$, or ${ }^{* "} \mathrm{P}<0.01$.

Fig. 4. Effects of Neo1 silence on the GC cell viability, cell motility and adhesion ability. Neo1 was suppressed by transfection with si-Neo1 in SGC-7901 cells, and then the effects of Neo1 silence on the cell viability, cell motility and adhesion ability were determined by CCK-8 assay, wound healing assay, and adhesion assay, respectively. The results showed that silence of Neo1 significantly inhibited the cell viability (A), cell motility (B) and adhesion ability (C). The potential underlying mechanism of cell motility and adhesion ability were further explored by analyzing the EMT process. EMT was induced by addition of TGF- $\beta 1$ to the cells. Both the mRNA and protein levels of the key proteins in the EMT process (E-Cadherin, N-Cadherin, and Vimentin) were measured by qRT-PCR and Western blot, respectively. The results indicated that silence of Neo1 inhibited EMT process (D-F) in GC cells. The experiments were repeated at least three times. Data are presented as the mean \pm standard deviation (SD). Neo1, neogenin-1; GC, gastric cancer; si, small interfering; NC, negative control; TGF, transforming growth factor; EMT, epithelial mesenchymal transition; qRT-

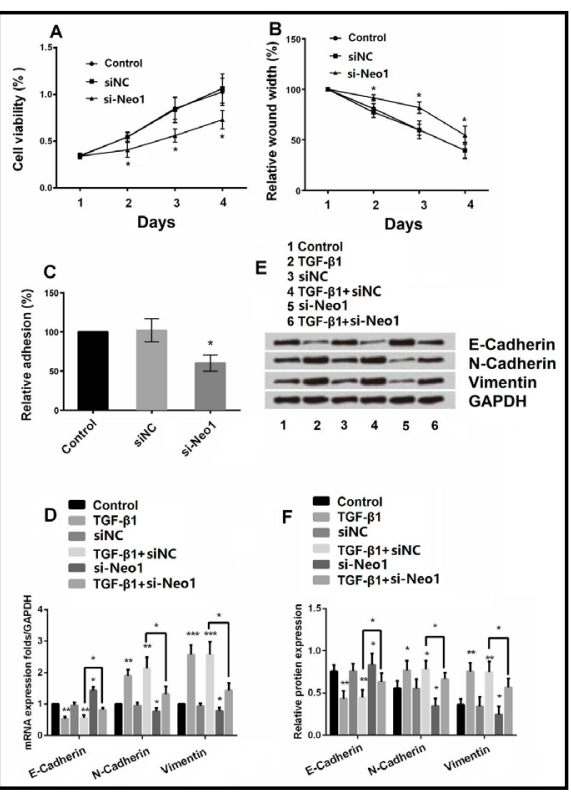
PCR, quantitative real-time reverse transcriptase polymerase chain reaction. Asterisk for the TGF- $\beta 1$ group indicated significance compared with control; Asterisk for the TGF- $\beta 1+$ siNC and the si-Neo1 groups indicated significance compared with the siNC group. ${ }^{*} \mathrm{P}<0.05$ or ${ }^{* *} \mathrm{P}<0.01$.

\section{Effects of Neo1 silence on GC cell viability, cell motility and adhesion ability}

Next, the effects of Neo1 silence on the GC cell viability, cell motility and adhesion ability were investigated. As expected, we found that silencing of Neo1 significantly inhibited the cell viability, cell motility and adhesion ability in GC cells (all $P<0.05$ ) (Fig. 4A-4C). In addition, the results showed that suppression of Neo1 significantly upregulated expression 
of E-Cadherin $(P<0.05$ for both mRNA and protein), and while statistically downregulated the expression of $\mathrm{N}$-Cadherin and Vimentin (all $P<0.05$ ). Similarly, the effects of Neo1 silencing on EMT in cells treated with TGF- $\beta 1$ were identical to that described above (all $P$ $<0.05$ ) (Fig. 4D-4F). In combination with the results from Fig. 3, our data implied that Neol may act as an oncogene by promoting the cancer cell proliferation and metastasis.

\section{Effects of Neo1 abnormal expression on ZEB1 level}

ZEB1 has been identified as an important inducer of EMT process, which is responsible for the increased aggressiveness and metastasis in a variety of human carcinomas [18]. Therefore, we analyzed the potential relationship between Neo1 and ZEB1. Both the mRNA and protein levels of ZEB1 were measured in cells abnormally expressing Neo1. Interestingly, the data revealed that both the mRNA and protein levels of ZEB1 were markedly upregulated by overexpression of Neo1 but down-regulated by silencing of Neo1 compared to their corresponding controls $(P<0.05)$ (Fig. 5 A-5C). The results suggested that Neo1 positively regulated the level of ZEB1.

\section{Effects of Neo1 abnormal expression on the Rac1/PI3K/AKT pathway}

We further explored the underlying pathway concerning the effects of Neo1 abnormal expression on EMT process in GC cells. The PI3K/AKT pathway has been reported to be

Fig. 5. Effects of Neo1 abnormal expression on ZEB1 level. The relationship between Neo1 and ZEB1 was investigated. Both mRNA and protein levels of ZEB1 were respectively determined by qRT-PCR and Western blot after overexpression or suppression of Neo1. Both the mRNA (A) and protein (B-C) levels of ZEB1 were markedly enhanced by overexpression of Neo1 but inhibited by silencing of Neo1. The experiments were repeated at least three times. Data are presented as the mean \pm standard deviation (SD). Neo1, neogenin-1; si, small interfering; NC, negative control; pc-Neo1, Neo1 expression vector; ZEB1, Zinc finger E-box-binding homeobox 1; qRT-PCR, quantitative real-time reverse transcriptase polymerase chain reaction. ${ }^{*} \mathrm{P}<0.05$.

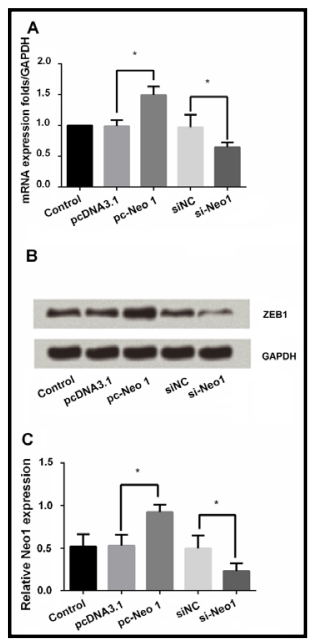

Fig. 6. Effects of Neo1 abnormal expression on key proteins expression in the Ras/PI3K/AKT pathway. The expression of key proteins in the Rac1/ PI3K/AKT pathway was investigated. The results demonstrated that Neo1 activated the Rac1/PI3K/ AKT pathway by increasing the expression of GTPRac1, p-PI3K, and p-AKT (A). The inhibitor of Rac1 (EHT1864) decreased the expression of GTP-Rac1, p-PI3K and p-AKT (B), whereas the inhibitor of PI3K (LY294002) had no significant effect on the expression of GTP-Rac1 (C). The expression of ZEB1 was markedly decreased by the inhibitors

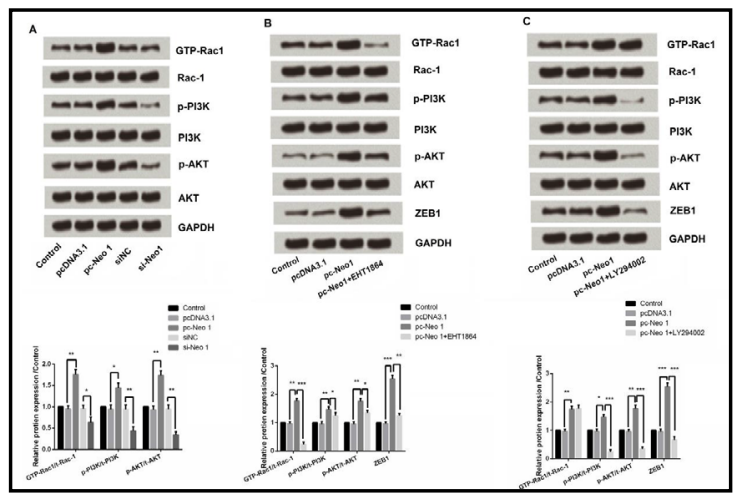
of Rac1 or PI3K. The experiments were repeated at least three times. Data are presented as the mean \pm standard deviation (SD). Neo1, neogenin-1; si, small interfering; NC, negative control; pc-Neo1, Neo1 expression vector; ZEB1, Zinc finger E-box-binding homeobox 1; GTP, guanosine-5'-triphosphate; PI3K, phosphoinositide 3-kinase. ${ }^{*} \mathrm{P}<0.05,{ }^{* *} \mathrm{P}<0.01$, or ${ }^{* * *} \mathrm{P}<0.001$. 


\section{Cellular Physiology Cell Physiol Biochem 2018;48:1457-1467 \\ \begin{tabular}{l|l} 
DOI: 10.1159/000492255 & $\begin{array}{l}\text { O 2018 The Author(s). Published by S. Karger AG, Basel } \\
\text { www.karger.com/cpb }\end{array}$
\end{tabular}}

Qu et al.: Neogenin-1 Acts as An Oncogene in GC

involved in the pathogenesis of many cancers, including GC [19]. The PI3K/AKT cascade is responsible for the GC cell apoptosis [20], proliferation [21], and metastasis [22]. Rac1 is a member of the Rho family of small GTPases, and PI3K has been considered as a downstream effector of Rac1 [23]. Hence, we analyzed the expression of the Rac1/PI3K/AKT pathwayrelated factors after changing the expression of Neo1. As revealed in Fig. 6A, the results showed that although the expression of Rac-1, PI3K, and AKT was not significantly changed by abnormal expression of Neo1, expression of their activated patterns including GTP-Rac1, p-PI3K, and p-AKT were significantly increased by overexpression of Neo1 but decreased by silencing of Neo1 compared to their corresponding controls $(P<0.05$ or $P<0.01)$. The results indicated that Neo1 activated the Rac1/PI3K/AKT pathway. To further confirm that Neo1 regulated the expression of ZEB1 by activation of the Rac1/PI3K/AKT pathway, we administered the inhibitor of Rac1 (EHT1864) and inhibitor of PI3K (LY294002), respectively. Our data showed that the inhibitor of Rac1, EHT1864, decreased the expression of p-PI3K and p-AKT compared to the pc-Neo1 group $(P<0.05)$, suggesting that the inhibitor of Rac1 partly inactivated the PI3K/AKT pathway. However, the inhibitor of PI3K, LY294002, had no significant effect on the expression of GTP-Rac1. It is noteworthy that both the inhibitors of Rac1 and PI3K significantly inhibited the expression of ZEB1 compared to the pc-Neo1 group $(P<0.01$ for EHT1864 or $P<0.001$ for LY294002) (Fig. 6 B and C). Taken together, our results implied that Neo1 positively regulated the expression of ZEB1 by activating the Rac1/PI3K/AKT pathway, leading to the promotion of EMT process in GC cells.

\section{Discussion}

In the present study, we found that Neo1 acted as an oncogene in GC. Overexpression of Neo1 reduced the GC cell sensitivity to cisplatin and increased the cell viability, motility and adhesion ability, and the silencing of Neo1 reversed the above results. Moreover, we observed that overexpression of Neo1 promoted EMT process by positively regulating the expression of ZEB1. Furthermore, we found that Neo1 regulated the expression of ZEB1 by activating the Rac1/PI3K/AKT pathway.

An increasing number of studies have demonstrated the critical roles of Neo1 in human diseases. Neo1 has been reported to be expressed in many tissues that shows active growth [14]. However, the functional role of Neo1 is complicated. The expression levels of Neo1 vary significantly among different cancers. For example, high expression of Neo1 was found in ESCC [13], moderate expression was displayed in pancreatic cancer [11], and low expression was revealed in breast cancer, colon cancer and glioma [7, 10,12]. Our study focused on the expression of Neo1 in GC. The data in our study were in line with a previous study showing high expression of Neo1 in different GC cell lines [14]. Nevertheless, the exact regulatory mechanism of Neo1 in GC is still not well understood. In order to address the problem, we altered the expression of Neo1 in GC cells by transfection with pc-Neo1 or si-Neo1, and then we analyzed the effects of aberrant expression of Neo1 on sensitivity to cisplatin, cell proliferation, and cell metastasis.

Resistance to chemotherapy has been a main obstacle for effective management of cancers [24]. However, the exact mechanism of chemo-resistance is still indistinct. Thus, understanding the mechanisms of resistance and strategies for overcoming resistance to chemotherapy is crucial for effective treatment of cancers. Cisplatin, in combination with other chemotherapeutic agents or on its own, is considered as an effective drug for treatment of various malignant tumors including GC $[25,26]$. In our study, we found that overexpression of Neo1 reduced GC cell sensitivity to cisplatin, and silencing of Neo1 elevated the sensitivity, indicating that the upregulated Neo1 may contribute to the sensitivity of cisplatin-resistant in GC cells. In addition, the results demonstrated that overexpression of Neo1 increased cell viability, motility and adhesion ability in SGC-7901 cells. It has been well established that EMT is involved in the early steps of metastasis and plays significant roles in cancer progression [27-29]. Therefore, we hypothesized that the functions of Neo1 on GC 
cells might be involved in EMT process. Previous studies have confirmed that TGF- $\beta$ induces EMT in different epithelial cells [30-32]. The hallmarks of EMT are the functional loss of adherens junction protein (E-Cadherin) and gain of mesenchymal markers (N-Cadherin and Vimentin) [33]. To confirm the hypothesis, we first induced EMT by administration of TGF- $\beta 1$. The low expression of E-Cadherin and high expressions of $\mathrm{N}$-Cadherin and Vimentin suggested that EMT was successfully induced. In the present study, overexpression of Neo1 significantly decreased the levels of E-Cadherin and elevated the levels of $\mathrm{N}$-Cadherin and Vimentin. Silencing of Neo1 reversed the results. This data implied that Neo1 aggravated the EMT process, leading to migration and invasion of GC cells.

The EMT process is regulated by several factors, such as EMT-transcription factors (EMT-TFs) zinc finger/homeodomain proteins ZEB1 and ZEB2 [34]. It has been suggested that ZEB proteins are highly expressed in many cancer cells including GC cells [35-39]. In addition, ZEB1 was identified as an independent prognostic factor of GC, and there is a significant correlation between ZEB1 expression and diffuse phenotype in GC [38]. Previous studies suggested that a variety of factors such as microRNAs (miRNAs) and ubiquitin ligase CUL4A can regulate the EMT process by modulating ZEB1 expression [40-44]. Our study provided new evidence that Neo1 promoted the EMT process in GC by regulating the expression of ZEB1. We further explored the possible signaling pathway with respect to the observed results. Activation of PI3K regulates cell growth, transformation, migration, and invasion $[45,46]$. The activity of PI3K is induced by the specific interaction of active Rac1 [47]. Moreover, Rac1 has been reported to activate AKT in a manner [48]. Additionally, EMTmediated motility and invasion have been revealed to activate the PI3K/AKT pathway in cancer cells $[15,49]$. In the present study, the results suggested that Neo1 could elevate the levels of GTP-Rac1, p-PI3K, and p-AKT, indicating that Neo1 activated the Rac1/PI3K/AKT pathway. Furthermore, administration of the inhibitor of Rac1 (EHT1864) decreased the expression of p-PI3K and p-AKT, while administration of the inhibitor of PI3K (LY294002) had no effects on the expression of Rac1. Our results were in line with previous studies showing PI3K was a downstream effector of Rac1 [45, 46, 50]. Interestingly, the expression of ZEB1 was significantly inhibited by both of these inhibitors, implying that Neo1 positively upregulated the expression of ZEB1 by activating the Rac1/PI3K/AKT pathway.

In conclusion, our data suggest that Neo1 functions as an oncogene in GC. Neo1 promotes cell proliferation, motility, and adhesion by up-regulation of ZEB1 via activation of the Rac1/ PI3K/AKT pathway in GC cells.

\section{Disclosure Statement}

The authors declare to have no competing interests.

\section{References}

1 Jemal A, Bray F, Center MM, Ferlay J, Ward E, Forman D: Global cancer statistics. CA: A Cancer Jr Clinic 2011;49:33-64.

-2 Ge L, Wang HJ, Yin D, Lei C, Zhu JF, Cai XH, Zhang GQ: Effectiveness of 5-flurouracil-based neoadjuvant chemotherapy in locally-advanced gastric/gastroesophageal cancer: a meta-analysis. World J Gastroenterol 2012;18:7384-7393.

3 Ohtsu A: Chemotherapy for metastatic gastric cancer: past, present, and future. J Gastroenterol 2008;43:256-264.

4 Wilson NH, Key B: Neogenin: one receptor, many functions. Int J Biochem Cell Biol 2007;39:874-878.

5 Wilson NH, Key B: Neogenin interacts with RGMa and netrin-1 to guide axons within the embryonic vertebrate forebrain. Develop Biol 2006;296:485-498.

6 Meyerhardt JA, Look AT, Bigner SH, Fearon ER: Identification and characterization of neogenin, a DCCrelated gene. Oncogene 1997;14:1129-1136. 


\section{Cellular Physiology Cell Physiol Biochem 2018;48:1457-1467 \begin{tabular}{l|l} 
DOI: 10.1159/000492255 & $\begin{array}{l}\text { O 2018 The Author(s). Published by S. Karger AG, Basel } \\
\text { www.karger.com/cpb }\end{array}$ \\
\hline
\end{tabular}}

Qu et al.: Neogenin-1 Acts as An Oncogene in GC

7 Vielmetter J, Kayyem JF, Roman JM, Dreyer WJ: Neogenin, an avian cell surface protein expressed during terminal neuronal differentiation, is closely related to the human tumor suppressor molecule deleted in colorectal cancer. J Cell Biol 1994;127:2009-2020.

-8 Milla LA, Arros A, Espinoza N, Remke M, Kool M, Taylor MD, Pfister SM, Wainwright BJ, Palma V: Neogenin1 is a sonic hedgehog target in medulloblastoma and is necessary for cell cycle progression. International J Cancer 2014;134:21-31.

-9 Song S, Mazurek N, Liu C, Sun Y, Ding QQ Liu K, Hung MC, Bresalier RS: Galectin-3 mediates nuclear betacatenin accumulation and Wnt signaling in human colon cancer cells by regulation of glycogen synthase kinase-3beta activity. Cancer Res 2009;69:1343-1349.

-10 Lee JE, Kim HJ, Ji YB, Kim SW, Park JS, Shin HJ, Han W, Kim SW, Kang KS, Noh DY: Neogenin expression may be inversely correlated to the tumorigenicity of human breast cancer. BMC Cancer 2005;5:154.

11 Link BC, Reichelt U, Schreiber M, Kaifi JT, Wachowiak R, Bogoevski D, Bubenheim M, Cataldegirmen G, Gawad KA, Issa R: Prognostic implications of netrin-1 expression and its receptors in patients with adenocarcinoma of the pancreas. Ann Surg Oncol 2007;14:2591-2599.

12 Wu X, Li Y, Wan X, Kayira TM, Cao R, Ju X, Zhu X, Zhao G: Down-Regulation of Neogenin Accelerated Glioma Progression through Promoter Methylation and Its Overexpression in SHG-44 Induced Apoptosis. Plos One 2011;7:e38074.

13 Hu YC, Lam KY, Law S, Wong J, Srivastava G: Identification of Differentially Expressed Genes in Esophageal Squamous Cell Carcinoma (ESCC) by cDNA Expression Array Overexpression of Fra-1, Neogenin, Id-1, and CDC25B Genes in ESCC. Clin Cancer Res 2001;7:2213-2221.

14 Kim SJ, Wang YG, Lee HW, Kang HG, La SH, Choi IJ, Irimura T, Ro JY, Bresalier RS, Chun KH: Up-regulation of neogenin-1 increases cell proliferation and motility in gastric cancer. Oncotarget 2014;5:3386-3398.

15 Larue L, Bellacosa A: Epithelial-mesenchymal transition in development and cancer: role of phosphatidylinositol 3' kinase/AKT pathways. Oncogene 2005;24:7443-7454.

-16 Kalluri R, Weinberg RA: The basics of epithelial-mesenchymal transition. J Clin Invest 2009;119:14201428.

17 Gupta GP, Massagué J: Cancer metastasis: building a framework. Cell 2006;127:679-695.

18 Salajegheh A. (2016): Zinc Finger E-Box Binding Homeobox 1 (ZEB1). In: Angiogenesis in Health, Disease and Malignancy. Springer, Cham.

19 Ito C, Nishizuka SS, Ishida K, Uesugi N, Sugai T, Tamura G, Koeda K, Sasaki A: Analysis of PIK3CA mutations and PI3K pathway proteins in advanced gastric cancer. J Surg Res 2017;212:195-204.

20 Li D, Qu X, Hou K, Zhang Y, Dong Q, Teng Y, Zhang J, Liu Y: PI3K/Akt is involved in bufalin-induced apoptosis in gastric cancer cells. Anticancer Drugs 2009;20:59-64.

21 Qu JL, Qu XJ, Zhao MF, Teng YE, Zhang Y, Hou KZ, Jiang YH, Yang XH, Liu YP: Gastric cancer exosomes promote tumour cell proliferation through PI3K/Akt and MAPK/ERK activation. Dig Liver Dis 2009;41:875-880.

22 Ho HH, Chang CS, Ho WC, Liao SY, Wu CH, Wang CJ: Anti-metastasis effects of gallic acid on gastric cancer cells involves inhibition of NF-kappaB activity and downregulation of PI3K/AKT/small GTPase signals. Food Chem Toxicol 2010;48:2508-2516.

23 Vanhaesebroeck B, Alessi DR: The PI3K-PDK1 connection: more than just a road to PKB. Biochem J 2000;346:561-576.

24 Camp ER, Jing L, Minnich DJ, Brank A, Moldawer LL, Mackay SL, Hochwald SN: Inducible nuclear factor- к B activation contributes to chemotherapy resistance in gastric cancer 1. J Am College Surg 2004;199:249258.

25 Kang YK, Kang WK, Shin DB, Chen J, Xiong J, Wang J, Lichinitser M, Guan Z, Khasanov R, Zheng L: Capecitabine/cisplatin versus 5-fluorouracil/cisplatin as first-line therapy in patients with advanced gastric cancer: a randomised phase III noninferiority trial. Ann Oncol 2009;20:666-673.

26 Boku N, Yamamoto S, Fukuda H, Shirao K, Doi T, Sawaki A, Koizumi W, Saito H, Yamaguchi K, Takiuchi H: Fluorouracil versus combination of irinotecan plus cisplatin versus S-1 in metastatic gastric cancer: a randomised phase 3 study. Lancet Oncol 2009;10:1063-1069.

27 Hollier BG, Evans K, Mani SA: The epithelial-to-mesenchymal transition and cancer stem cells: a coalition against cancer therapies. J Mammary Gland Biol Neopl 2009;14:29-43.

-28 Tania M, Khan MA, Fu J: Epithelial to mesenchymal transition inducing transcription factors and metastatic cancer. Tumor Biol 2014;35:7335-7342. 


\section{Cellular Physiology Cell Physiol Biochem 2018;48:1457-1467 \begin{tabular}{l|l} 
DOI: 10.1159/000492255 & $\begin{array}{l}\text { O 2018 The Author(s). Published by S. Karger AG, Basel } \\
\text { www.karger.com/cpb }\end{array}$ \\
\hline
\end{tabular}}

Qu et al.: Neogenin-1 Acts as An Oncogene in GC

29 Yang, Jing, Weinberg, Robert A: Epithelial-mesenchymal transition: at the crossroads of development and tumor metastasis. Dev Cell 2008;14:818-829.

-30 Xu J, Lamouille S, Derynck R: TGF-beta-induced epithelial to mesenchymal transition. Cell Res 2009;19:156-172.

-31 Katsuno Y, Lamouille S, Derynck R: TGF- $\beta$ signaling and epithelial-mesenchymal transition in cancer progression. Curr Opin Oncol 2013;25:76-84.

-32 Zhou Q Zheng X, Chen L, Xu B, Yang X, Jiang J, Wu C: Smad2/3/4 Pathway Contributes to TGF-beta-Induced MiRNA-181b Expression to Promote Gastric Cancer Metastasis by Targeting Timp3. Cell Physiol Biochem 2016;39:453-466.

33 Latifa B, Wagner EF: News and views on EMT Fra-1 controls EMT in mammary epithelial cells. Mol Cell Oncol. 2016;3:e990307.

-34 Thiery JP, Acloque H, Huang RY, Nieto MA: Epithelial-mesenchymal transitions in development and disease. Cell 2009;139:871-890.

35 Dohadwala M, Yang SC, Luo J, Sharma S, Batra RK, Huang M, Lin Y, Goodglick L, Krysan K, Fishbein MC: Cyclooxygenase-2-dependent regulation of E-cadherin: prostaglandin E(2) induces transcriptional repressors ZEB1 and snail in non-small cell lung cancer. Cancer Res 2006;66:5338-5345.

-36 Fang Y, Wei J, Cao J, Zhao H, Liao B, Qiu S, Wang D, Luo J, Chen W: Protein Expression of ZEB2 in Renal Cell Carcinoma and Its Prognostic Significance in Patient Survival. Plos One 2013;8:e62558.

-37 Herrmann P, Aronica SM: Estrogen and tamoxifen up-regulate FXYD3 on breast cancer cells: assessing the differential roles of ER $\alpha$ and ZEB1. SpringerPlus 2015;4:1-8.

38 Yabusaki N, Yamada S, Murai T, Kanda M, Kobayashi D, Tanaka C, Fujii T, Nakayama G, Sugimoto H, Koike M: Abstract 4101: Clinical significance of ZEB1 mRNA levels in peritoneal washing for gastric cancer. Cancer Res 2015;75:4101-4101.

-39 Okugawa Y, Toiyama Y, Tanaka K, Matsusita K, Fujikawa H, Saigusa S, Ohi M, Inoue Y, Mohri Y, Uchida K: Clinical significance of Zinc finger E-box Binding homeobox 1 (ZEB1) in human gastric cancer. J Surgic Oncol 2012;106:280-285.

40 Larsen JE, Nathan V, Osborne JK, Farrow RK, Deb D, Sullivan JP, Dospoy PD, Augustyn A, Hight SK, Sato M: ZEB1 drives epithelial-to-mesenchymal transition in lung cancer. J Clin Invest 2016;126:3219-3235.

-41 Wu X, Xiong E, Wang W, Scali M, Cresti M: Universal sample preparation method integrating trichloroacetic acid/acetone precipitation with phenol extraction for crop proteomic analysis. Nat Protoc 2014;9:362-374.

42 Brabletz S, Brabletz T: The ZEB/miR-200 feedback loop-a motor of cellular plasticity in development and cancer? EMBO Report 2010;11:670-677.

43 Majid S, Dar AA, Saini S, Deng G, Chang I, Greene K, Tanaka Y, Dahiya R, Yamamura S: MicroRNA-23b functions as a tumor suppressor by regulating Zeb1 in bladder cancer. Plos One 2013;8:e67686.

44 Gregory PA, Bert AG, Paterson EL, Barry SC, Tsykin A, Farshid G, Vadas MA, Khewgoodall Y, Goodall GJ: The miR-200 family and miR-205 regulate epithelial to mesenchymal transition by targeting ZEB1 and SIP1. Nature Cell Biol 2008;10:593-601.

45 Rajalingam K, Schreck R, Rapp UR, Albert S: Ras oncogenes and their downstream targets. Biochimica Et Biophysica Acta Molecular Cell Res 2007;1773:1177-1195.

-46 Charvat S, Le GC, Chignol MC, Schmitt D, Serres M: Ras-transfection up-regulated HaCaT cell migration: inhibition by Marimastat. Clin Exp Metastasis 1999;17:677-685.

47 Bokoch GM, Vlahos CJ, Wang Y, Knaus UG, Traynor-Kaplan AE: Rac GTPase interacts specifically with phosphatidylinositol 3-kinase. Biochem J 1996;315:775-779.

-48 Genot EM, Arrieumerlou C, Ku G, Burgering BM, Weiss A, Kramer IM: The T-cell receptor regulates Akt (protein kinase B) via a pathway involving Rac1 and phosphatidylinositide 3-kinase. Mol Cell Biol 2000;20:5469-5478.

49 Kang MH, Kim JS, Seo JE, Oh SC, Yoo YA: BMP2 accelerates the motility and invasiveness of gastric cancer cells via activation of the phosphatidylinositol 3-kinase (PI3K)/Akt pathway. Experimental Cell Res 2010;316:24-37.

50 Castellano E, Downward J: RAS Interaction with PI3K: More Than Just Another Effector Pathway. Genes Cancer 2011;2:261-274. 